BglaGluR1 . 1 BglaGluR1 BglaGluR2 BglaGluR3 BglaGluR4 BglaGluR4. 1 BglaGluR6. 1 BglaGluR6 BglaGluR7 BglaGluR8 BglaGluR8. 1 BglaGluR9.1 BglaGluR9.2 BglaGluR9 BglaGluR10 BglaGluN11 BglaGluN12 BglaGluN13 BglaGluN14 BglaIR25a BglaIR8a BglaIR1 BglaIR2 BglaIR3 BglaIR4 BglaIR5 consensus

BglaGluR1.1 BglaGluR1 BglaGluR2 BglagluR3 BglaGluR4 BglaGluR4.1 BglaGluR6. 1 BglaGluR6 BglaGluR7 BglaGluR8 BglaGluR8. 1 BglaGluR9. 1 BglaGluR9.2 BglaGluR9 BglaGluR10 BglaGluN11 BglaGluN12 BglaGluN13 BglaGluN14 BglaIR25a BglaIR8a BglaIR1 BglaIR2 BglaIR3 BglaIR4 BglaIR5 consensus
MDLNLDRDFFAQFIYGGVDITAFQFLNNRSET . YRKWQQIWEQ . . MDLNLDRDFFAQFIYGGVDITAFQFLNNRSET . YRKWQQIWEQ YKDQYPDLFPLKTGSALMMDAVLLL HKALTSDLQKHKSLKENAVRTCD . MKLLVDTGHWFQIDAALAGDAFRLFSSITNT . YFIPDRHSEL . GDLGMLEMNLTNFKIGGVNITGFQIVDPYNTT . SELFLSTWAN . GIPLIGRFRFIGYCSDLAEMVSRNVGYEYHIR. FVKDGEYGRK

RDVVMNVLQA AQQI HLLHDKIDWFVTYPDFED. FLSENTTWSG TLYGLQLLREEK IPQN ISDVVDLHNVTMSRLDLATA IDVVGLLRHILWKEAPGCNRERLLD KLISVRNHYFTTFVTVLNPQHTRLVLDEALSLSLFSTPHKWLL. VNLGLREYDLSRFVDSYANVTVIRLMMDYNSMYCKLQHDY INLRRA VFHDA IMLYRQMNGS ITLDLGLLDLEDYRYGSAN ITAYRL IDPARDK. VVNVKTDWLR . . . . HAKKGHESPLMGYAEIEGNSSHTLLTETALAYDAYELFAYALMAYSKVQEVATMNQPC

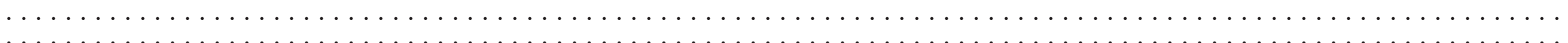

RDNLIRKVLVKARSYGMLHQPDMTFFFMDPASYYEPFSGASKM. ETVNILLDQARDLAMLSMPPYGWLFYDPGDDI.KILFESYRDIS

MVVGRSKSVRWSVKVRCSSG QSNTSVRWSV

ESVDFQTHFS CNVQSQA TNQSARSLYWKYGEHLYKKVEQSTSKSTDSRGGRFPLKT SPF S SKRD IDFGF SKSRD I ARESRFEGF TRKPLFRDRLSGPLTGPNDLD DGETIYRHMLNVSIPGETHIKFNQNGTLKQTD. I IWNLQWLG. . EGRA ILSRFQTLAEEVNIRVNAEKIYHDAELMGMTGEDWAWIV

\section{... MWINEGFQMVKCPQVQTQ} GSKNTTEWKEVGRWTVNGLKMS EITWPGGGSTPPSG

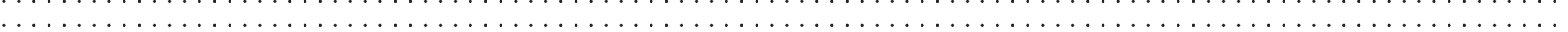
$\ldots$

ASSSRYSDGGSSL

LSALNG.ETTWQSSD

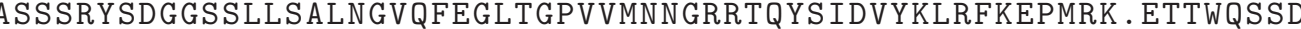
YNNGTKGIQCWRDP PVPWMHGPA IRDALLKHGVRVNTKLDIMHLEYRTTLRS . IGTWEHG. DSDVKTGHGHVVLEEMKKTKFEGVTGHVAFNEFGQRKEFTLDVYNVAMTRGTAK. IGYWNEKE. QKDGTWNGVIGELIKHPLLLLLSSVALGDSY. GHMGD SYGHMGDSYS SMCDSYGHMGDSYGHMGD SYGHMGDSYSHMGDSYG MDGKSGNINFHATGQR INYSVDVQEVTMYKGISI. LGTWHSRD . GFNVYGEEQVLDKA. NRTIDNRTRIVTSIDEEPY DHIVSA EAFTWQLKNT TSPYNGALGQY IWDKS SKGRTNFT IDILRYIGYTKQEI. GQVVF ING . . TADVTLSQFNQTRA. VLAEPLEMKK IKLRVATQQQDPF I VGGGQKTEKGKMRQTVRRLEMDGCTGHLDFSVQGQRQESFLQLMTLEGYKTGE . SGTWRSKQSELKQRVEPSRSYSTVSRLE . GNVFGNEPLRVTVMIEDPFV DKMYTWRHGNSLLNYMKSSA IEGLSGLIKYDG. GERMDFVLDLLSLTPKGLEV . . VGKWDRKH . . . . . . . RLNITRENVTPTDTKITNKKLIVTTYITKPYV $\ldots \ldots \ldots \ldots \ldots \ldots \ldots \ldots \ldots \ldots \ldots \ldots \ldots \ldots \ldots \ldots \ldots \ldots \ldots \ldots \ldots \ldots \ldots \ldots \ldots \ldots \ldots \ldots \ldots \ldots \ldots \ldots \ldots \ldots \ldots \ldots \ldots \ldots \ldots \ldots \ldots \ldots$

\section{MSLFNELK RGYWYSEP} SNKDVIDNEKFLRVIKETNFTDGRTGKIYFDKNGQRKNFHLYLYDHGGEESMYKTIARWGPNG
NSLTDISLKFWMNRVLRNSNVTGLTGMFQFNAHGRRNNFYLTLTSFTGVENYL. RGYWYSEP

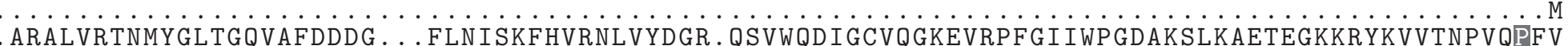
PDNPDNPEGTENRDLDLNDDPDASSGSSSYKNSSRKYPKKI GNSKMKPYGKNDV. KRRYAEKHRRPRRQLS. DKFSLN ILQKNCWQTVSESN IRNVCEQVKDKYG KPKRAFLRI ATLYEEPYVIYRQPEEEGKCDDKSLPCH IYQRNEKKVLLVETLFF. VGRWTVNG . . . . . LKMSEITWPGGGSTPPSGKPKRAFLRIATLYEEPYV KPKRAFLRIATLYEEPYV IYRQPEEEGKCDDKSLPCH IYQRNEKKVLLVETLFF.VGRWTVNG . . . LKMSEITWPGGGSTPPSGKPKRAFLRIATLYEEPYV
NESESWSNGDDVYNALKATKIEGETGQVSFNEKGDRVNPMYEIMN INANRRAMS.VGLFGHKDQA IGLRMMGNNLTWPGNVHVKPKGEKISRNLTIVTLVEKPFV

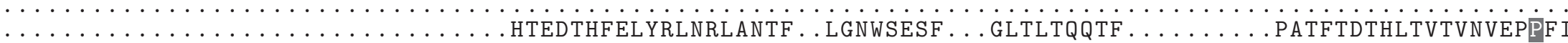

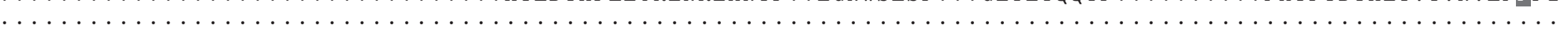
MDTRHWSVLLLHFAALLTEIH. TETSKPD . . . TPTPPPNTTTTTVTTTTGSSTTAGPKKLIAITLLDPPFV MHGNSLYDALREVSFQGYSGHVLFDIHGRRQNYSIDVLSLTSGSSLRK. VGQWHSLS . . . GLWLDREEKVKDQV . . . RPDMRDNRTVI ISTLQVPFLM MATTLAPA ANHLVISVVDQEPFI 
BglaGluR1 . 1 BglaGluR1 BglaGluR2 BglaGluR3 BglaGluR4 BglaGluR4 . 1 BglaGluR6. BglaGluR6 BglaGluR7 BglaGluR8 BglaGluR8. 1 BglaGluR9. 1 BglaGluR9. BglaGluR9 BglaGluR10 BglaGluN11 BglaGlun12 BglaGluN13 BglaGluN14 BglaIR8a BglaIR1 BglaIR2 BglaIR3 BglaIR4 BglaIR5 consensus BglaIR25a

MRIENRNGAPPM . MRIENRNGAPPM . MRKCPPDGTPC . HMGD SYGQM

SKRTDS .

MKDNVT .

\section{SLKRDHMLLKGN .}

IRRSEP ILG

SLKRDHMLLKGN .

DKENPTPD .

MTEAPHADYGTCLSDTPCLNLTNKVKSQEVVWQA IEAYENGSTAESGLFV

LYTDLQDMSGNQTLD .

IYRQPEEEGKC .
MISKT IRDGTPK

MAALEVTDGIPL $\ldots \ldots \ldots \ldots$

MI

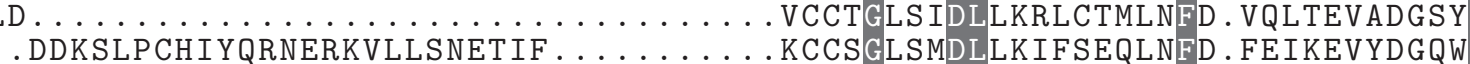

LDLEGYCIDLIEALARSELFE. YQVYLADD . Y Y LDLEGYCIDLIEALARSELFE. YQVYLADD . Y Y EQFEGYCVDLLKKISVLLKFD . YA IRLVADRNY EPVEGFCIDLTKAVAQKVGFD . YIIQFVKDGSY . GDSYSHMCDSYGHMGD SYGHM . GDSYGHMGDSY FRF IGYCSDLAEMVSRNVGYE. YH IRFVKDGEYG NTYSGFSYDLIQK ISENTRYE. FELYETEDDDVK GDFEGFCIDILEEVSR ILGFR . YNISKVPDGKYG GQYYGFCIDLLNEIAKTRNFT . YEIQIESKVGKR NRFEGFA VELI AD I AEMLDFE. YEIYLVNDGKFG DYYEGFS IDLIKRLAQELKFK . YELYVSPGNVYG NRFEGFA VELI AD I AEMLDFE. YEIYLVNDGKF ELYEGFCIDLTKELARIVGFN . FRIELVPDGNYC KCCSGLSMDLLK IFSEQLNFD.FEIKEVYDGQW . DKNETDGT . WNGI I GQL . DKNETDGT . AR. SENGS AS. FPNGT . HMGDSYGH RK.QKDGT .VK SYKTHGWTGMTNET I GNNNNWDGVMGAL

AK L LSNGS . . NKLPNGE AKNLLTNQ NKLPNGE . SPNTQGE QR . SSNGT .SPINNNES .L . . LVNKT FRNTSEP GSYYGYSMDVLTEIAKTVGFT FTVRECDEGGYG . . MLENGI W W $\dot{W} \dot{C} \dot{I} \dot{G N}$ $\ldots \ldots \ldots \ldots \ldots \ldots \ldots \ldots \ldots \ldots \ldots \ldots \ldots \ldots \ldots \ldots \ldots$

MHEDR

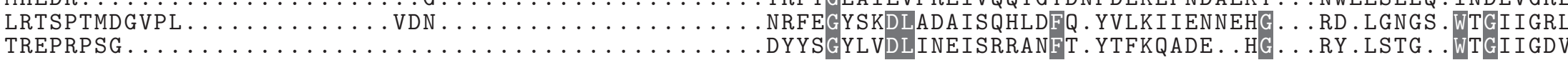
G TRFTGLA IEVFREIVQQTGYDNFDLKLPNDAEKY ... NWEESLLQ. INDLVGRL DYYSGYLVDLIN
DMKLANAL A KEVGFT . HEFR IVADNA Y

210

BglaGluR1. 1 BglaGluR1 BglaGluR2 BglaGluR3 BglaGluR4 BglaGluR4.1 BglaGluR6 . BglaGluR6 BglaGluR7 BglaGluR8 BglaGluR8.1 BglaGluR9. 1 BglaGluR9.2 BglaGluR9 BglaGluR10 BglaGluN11 BglaGluN12 BglaGluN13 BglaGluN14 BglaIR25a BglaIR8a BglaIR1 BglaIR2 BglaIR3 BglaIR4

BglaIR5 consensus

ISQERDIA . . . . VAPLTITQDRERVVDFTKPFMDT ISQERDIA . . . . . V VAPLTITQDRERVVDFTKPFMDT LTGKADMA . . . . I IAALTITEIRERFVDFSKPFMNL . VRHEADMA . . . . . IAPFTITADRSRVIDFTKPFMSL GDSYGDMGDSYGHMAPLTI TSDREKVLDFTKPFMSL IKHDADIA . . . . . IAPLTI TSDREKVLDFTKPFMSL VSGNASMA . . . . I IGALEVTAEREKLISFSYTVMSS VRSRADLG . . . . . VGAFQITPERAGAVDFTKPYITK VNRKADIG . . . . IGDLTINLVREQAVDFTKPFLTL MEI ISGVADLA LSGNATLA . . . . . CGA ISI TSARETV IDFSLGVVST . LAGNATMS . . . . VAPLSINAQREEA IDFTKPFKTR IDRRADLA ..... IAPLTITYLREQVIDFTKPFLNL TDNTAHFA . . . . VAAFSITNQREVA IDFTDPYFFS I HKKADMA . . . . I I GALSI TPERSNA VDFSMPFLQT LTKEADIV . . . . MTSFKINPDRASAVNFSVPYLET . MRQEADLI .....V.VAPLTINPERANDIDFTKPFKYQ . RADIA . . . . VGPISVMNERETVIDFTVPYYDLV ...EADVI.

LGALTVTAERDSVIDFTLPYYDF

RSNEADVA .... IGAFTLTPNLASEVQVSQPILHT

IDKTADMA . . . . IGPLSITLEREKVVDFSMSFMKS VKGEAQIG.

GGALTVTTKREEAVDFTKPYLSN
ISIMIKKPDKTKPG. . VFSFMDPLDTRV

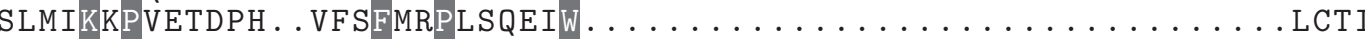

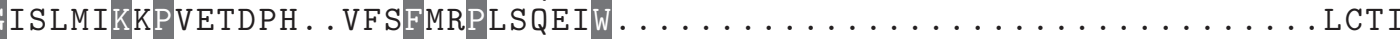

YFQFLGPFSGELW

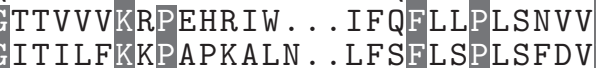

GVT IVIKKPQSQRPG . . VF SFMEPLSLEV

VTIVIKKPQSQRPG.

Y ISVLMK IPTRETS . . . YFEFLNPLSPVV

IFQFMMPFSLEL

YFEFLNPLSPVV

IS ILFKVPRKEKPG . . LF SFLNPLA IEIWLYVIGESEFYTLA IEIWLYVIGESELYTLAMEIWLYVI

MLAFLEPFSTKV

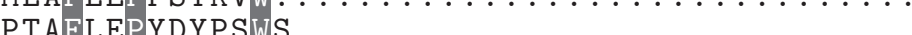

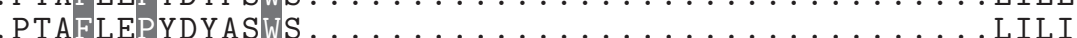

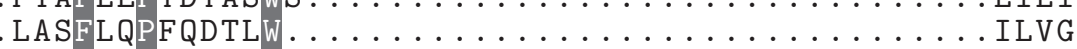

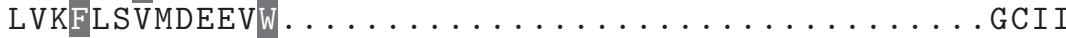

LFYYIDVFSTQA

MLEPFATEVW

MYPFGIEV

INI IKYPS

GYKLLYKIPDSWHPGEAMVTLLRPF SPGL
GISALLQQPAKVRPN . . MF SFMEPFSLALI

SVNLLVQKPTWEDLG. . LGYLVRPFSADYW .

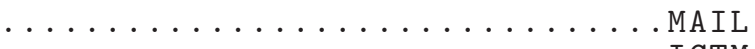


BglaGluR1.1 BglaGluR1 BglaGluR2 BglaGluR3 BglaGluR4 BglaGluR4.1 BglaGluR6 BglaGluR7 BglaGluR8 BglaGluR8.1 BglaGluR9 2 BglaGluR9 BglaGluR10 BglaGluN11 BglaGluN12 BglaGluN13 BglaGluN14 BglaIR25a BglaIR8a BglaIR1 BglaIR2 BglaIR3 BglaIR4 BglaIR5 consensus BglaGluR9.1
I GFLAVS FAFIGVSVVLFLVS LF I MVAGLALYVMSRFDP . . . IAFVSTSLMLFAVSRVN AAYLCVSEMLFVIARFSPYEWCNPHP VAYLTVSI GLFLVSRF SPVEWKKVREHQNN IDWTQVRADKDGLYHNDFS LFNSFWFS VAYLTVSI GLFLVSRFSPVEWKKVREHQNN IDWTQVRADKD GLYHNDFSL .

CAFVIVSV I L Y I ERVGRS KDTQEVEI TERERERRVRRVKREREKSKRGSKRERERRVREERERDK

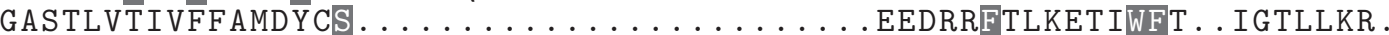

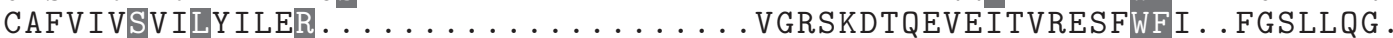
GAYLIVSFTIFTLARFSPYEWYNPHP . . . . . . . . CNPDTDVVENTFNLSNSLWFT . VGTLMQQ. VSAHITAVCMALFEWNSPFG . . . . . . . . . . LNPWGRKRNKNYSLASGLTMV . . FSVLFGH . FSVHATGASIFIFEWLSPYGL . . . . . . . . . . DQGKTSLRVKTCELQI FSVHATGSSILIFEWLSPYGI

LSVHVVALVLYLLDRFSPFGRFK NRGLTHMRDHKFSLFRSFWLI . . WAMLFST . . LAKSDDTEEDALNLSSAMWFS . . WGVLLNS .

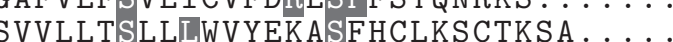
SVLLTSLLLWVYEKASFHCLKSCTKSA . . . . . . . . GDVKADNLGQTFYLGKSPVLAFNNNTFVSKKPMPQERAP

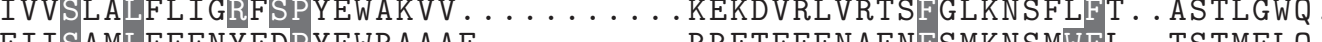

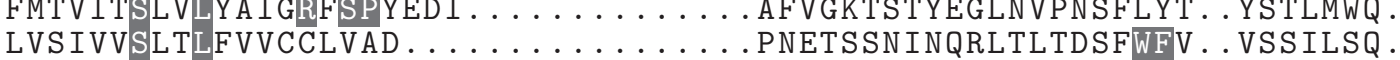
VVLLLIGIVFFVIGKFSPYEWGNVA . . . . . . . . ADRDPRGAKNSFTLRNSYLFA . LSTITWQ. IGFLAVSFVIFVGRE LGFIGVSTVLYLVGR FAYIGVSVVIFIVSRF FAFIGVSVVLFLVS

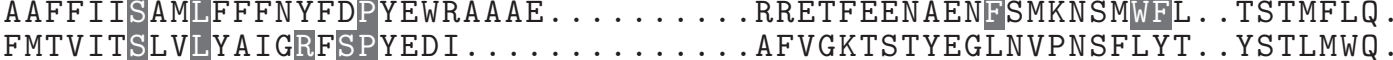

C. STPRS

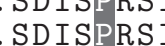

SDIYPRSI

. CDISPRSI

TDYSPRTSMRAIIAF.

S. LGSPHA ISSRIVSSA.

G. CEIAPRALSTRLVAGM.

. SDTCPRS ISGRI I GGA SISGRI I GGA. SNTDSTPTTIPGRILTSA. G. TDFAPVPISQRILTAG N. TDSTPTTIPGRILTSA.

G. SDINPRAVSTRLVGDIFYFVAFLSPFVA TVKTKSPKAWPSKVMQNF SVRTS S. VRFLAN I SVTDSPKIASRFLANI

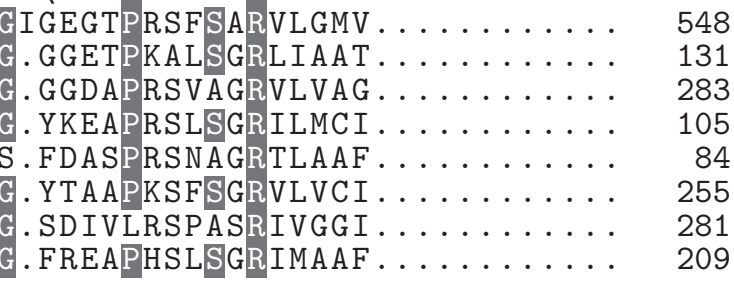

BglaGluR1.1 BglaGluR1 BglaGluR2 BglaGluR3 BglaGluR4 BglaGluR4.1 BglaGluR6. 1 BglaGluR6 BglaGluR7 BglaGluR8 BglaGluR8.1 BglaGluR9.1 BglaGluR9.2 BglaGluR9 BglaGluR10 BglaGluN11 BglaGluN12 BglaGluN13 BglaGluN14 BglaIR25a BglaIR8a BglaIR1 BglaIR2 BglaIR3 BglaIR4

BglaIR5

consensus

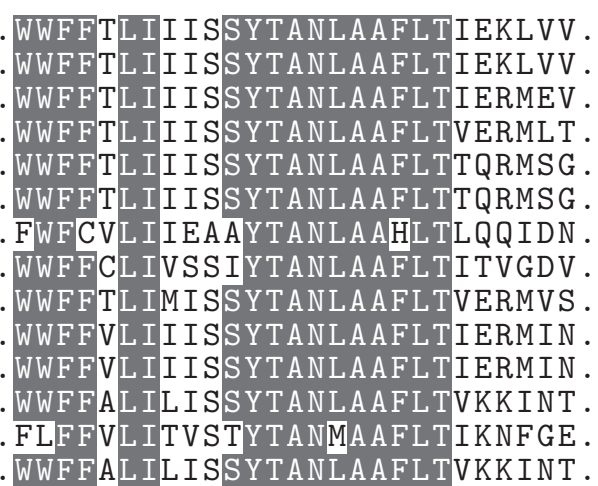

SISSADDI. SISSADDL PINSADD PIESAED SIRSAED SIRSAEDL RIKTVHDL DMNSAAD DINSADD PIESADDL PIESADDI PIKSVTDI TVNSFET PIKSVTD PIESAED NYYDIQD . PRLQDIRIGLLPGSAVHDSLKN WWFFTLIIISSYTANLAAFLTVERMVS. WAFAA IF I IASYTANLAAFIAGKHAG I . .

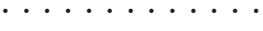
ALFAVVFCASYTANL A AFMITKDDYY. DLSGIQDYRLQNPYTMNPPEKYATIPNGSTEANTRT

VGHPTIKYGTKRSGTSWRFFE. . VGHPTIKYGTKRSGTSWRFFEK . VKQTEIKYGPYRGGSTYMFFNQ ... TTVSLYQRMWSFMTS . QPDV AGQSSLMYGVERGSDLHEFLQN . . TKEDPYERLWAF IKLNEDKV ATQNIFDYGTVDGSQTAYFFEH AKQSKIKYGLYGGGATQEFFEK LNHPSIKYGAVTSGSTLQFFLN . . . SDVPVYRQMGEYMKT . . . SDVPVYRQMGEYMKT HSEV . TNIEHFAKMWAQMSEIEPGS AESKKIGVSTVINSATMAFLKN DQGQKNVYKKIWSKVLG SNG AQQTK IKY GTVKD S G IMSFFKN . . . TN IEHFAKMW AQMSEIEP GS AKQTEI . DLSGIQDYRLQNPY TLVADRY NYKA I HSYMKN

NHIDMYNYMKAYNLP WWLFGFII IATYTANLA AF LTVSRLET . . . . PIESLDDL . . . . . . SKQVKVQYAPENGSMAMIYFRRMADIESMFYE. IWKNMTLKSEN I FWFAIIIMSTFTANLAAFLTVSRMGA. TLPADYED. VDNKDVK IGAMKYHHVYQVLKHGKVSHNN IFSKLFVYIDD . SQE LDQTEVAFGTVEKGSTFYFFKK . . . SSVPEYQRLWQHMNTRVPSP CRQNKVDMLIVANSSSFNYLSN . . . SKRLLERRLYQKLKP . . ENV $\ldots \ldots \ldots \ldots \ldots W$. . . . . . . . . . YTKTAEEL $\cdots \cdots \cdots \cdots$ WWLFS IMTLASYIAALSVLLFRVPEIR . . TLPFSNMDEF

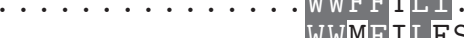

WWMFILFSLIAYTANLTAYFLARPEQIPKM . PFKTYEDI $* * * * * * * * * * *$ LADTNIRVGVLLSGSTESLLRN . . SRSETLRSIYSKINA . Q QNT ALFALVFLASYTANLAAFMITKEEYY 
BglaGluR1.1 BglaGluR1 BglaGluR2 BglaGluR3 BglaGluR4 BglaGluR4.1 BglaGluR6. BglaGluR7 BglaGluR8 BglaGluR8.1 BglaGluR9.1 BglaGluR9. 2 BglaGluR9 BglaGluR10 BglaGluN11 BglaGluN12 BglaGluN13 BglaGluN14 BglaIR25a BglaIR8a BglaIR1 BglaIR2 BglaIR3 BglaIR4 BglaIR5 consensus
VRESKSTYAFLLESAMNTYYGQQEP LFSEYHEGVKKVRESKSTYAFLLESAMNTYYGQQEP FVSSTNEGIQRVRDSKGKYAFLLDSTFNEYHNQRKP FVRTHEEGIQRVRQSNGKYAYLTESLTIDYVSNRKP FVKDNDEGIKRVRESNGQYVLP

FVKDNDEGIKRVRESNGQYVYLIESTLNEYYSSRLP LLDNTTEI INRVKA . . GTMAFIADGVTNGYYANQH . MVRNVDKGFARVNQ . . GGYAFIWDSPVIRHKISND . FVKSLDEAIQKVKK . GDYAYITESTTAEFVIHRH LVQSNEEGIERVLKSKGKYAFLAESSFIEYINERSP LVQSNEEGIERVLKSKGKYAFLAESSF IEYINERSP MVDNTTEGFKKVME . . . . . . . . . . .

QVVNASEGMKKVKK . . GTHAFIFDYLINEAFQNRH . . MVDNTTEGFKKIKT . . DDYAFFWDTTVNKFQTLID . PVPKTDEGIDWVMP. . DISLKLSP YPQTSVKDA IRALKNQEIQAF IYDSTTLEYEFGKDVGCKLKTVGKR I AETGYGLAFPKK . . . . SQWV ... DVESGITALKQGK. IQAF IYDSSVLEYRA SRDPKCGLVTVGNRYAMTGYGVGFPPNNKQFKNPWI A I ADIRLG . . . . . . . . ELQAF IWDSPRLEYEASND . CDLTTAGDLFGRSGLGIGLPKN . . . . SPWT GSIDKAQLAVWDYPVSDKYTKLWDTMNKNTFP . . . VVNVEAAIKRVVNEDFAFIG . . . . . . . . TNQDSLAVWEYPLETLSDLDL A SVSLRNSHFTPTHW. LEAVQHFEALTPNCNKYG . . . . . . . . T I I WA WIKHNKEGVQRVKDSGGKYVLLMETVKADYVAATN . .CDVIPYGETLSTFGYSFAGQKK . . . . . SPLM WVND IEDGI KRVRESNGYYAF I GEA GELSF I A SKRP.CDLLVSGTYITRTTYALAVQKD . . . . SPLR FVGD IDKAVKKMMA AD GKL A LFLESS I AQYLATQDP . CDKMVIGERLGDHS IGF I CQKN . . . . STVC

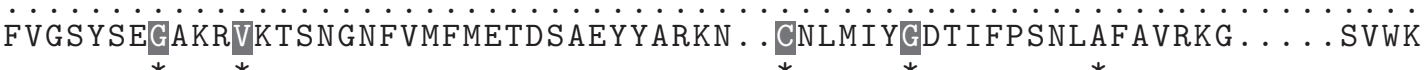

